in vivo $33: 2235-2240(2019)$

doi:10.21873/invivo.11728

\title{
Distribution of Solitary and Multiple Enchondromas of the Hand
}

\author{
SHINJI MIWA ${ }^{1,2}$, HIDEKI OKAMOTO ${ }^{1}$, SATOSHI YAMADA ${ }^{1}$, YOHEI KAWAGUCHI ${ }^{1}$, \\ KOJIRO ENDO ${ }^{1}$, HISAKI AIBA ${ }^{1}$, KATSUHIRO HAYASHI ${ }^{1,2}$, HIROAKI KIMURA $^{1,2}$, \\ ISATO SEKIYA ${ }^{3}$, TAKANOBU OTSUKA ${ }^{1}$ and HIROYUKI TSUCHIYA ${ }^{2}$ \\ ${ }^{1}$ Department of Orthopedic Surgery, Graduate School of Medical Science, Nagoya City University, Nagoya, Japan; \\ ${ }^{2}$ Department of Orthopedic Surgery, Graduate School of Medical Science, Kanazawa University, Kanazawa, Japan; \\ ${ }^{3}$ Department of Orthopaedic Surgery, Kainan Hospital, \\ The Aichi Prefectural Federation of Agricultural Cooperative for Health and Welfare, Yatomi, Japan
}

\begin{abstract}
Background/Aim: Although some patients with enchondroma have multiple lesions, no study has investigated the distribution of lesions in patients with multiple enchondromas. Patients and Methods: This retrospective study included 118 patients with enchondroma of the hand. The incidence and characteristic feature of multiple enchondromas of the hand were investigated. Results: Four patients (3.4\%) had multiple enchondromas. In all the patients with multiple enchondromas, the lesions occurred in the middle phalanx, proximal phalanx, and metacarpal bone in the same digital ray. Conclusion: The development of the hand rapidly progresses from intrauterine day 33 to day 54. The digital rays are evident on intrauterine day 41, and separation of the distal phalanx, middle phalanx, proximal phalanx, and metacarpal bone is completed until intrauterine day 54. The successive occurrence of multiple enchondroma lesions in the same digital ray in all four cases suggests that the occurrence of lesions preceded the separation of the hand bones and the lesions were divided during the development of these bones.
\end{abstract}

Enchondroma is the most common bone tumor of the hand. It usually occurs in the proximal phalanx, middle phalanx, and metacarpals (1-3) and causes thinning of the bone cortex

This article is freely accessible online.

Correspondence to: Hideki Okamoto, Department of Orthopedic Surgery, Graduate School of Medical Science, Nagoya City University, 1 Kawasumi, Mizuho-cho, Mizuho-ku, Nagoya, 4678601, Japan. Tel: +81 528518236, Fax: +81 528420266, e-mail: yands53okamoto@yahoo.co.jp

Key Words: Enchondroma, hand, distribution, multiple.
(4). Radiographs and computed tomography (CT) imaging show local osteolytic lesions with calcification and localized scalloping of the cortical bone. Magnetic resonance imaging (MRI) shows intermediate signal intensity on T1-weighted images and high signal intensity on T2-weighted images for rich cartilaginous areas and low signal intensity on both T1and T2-weighted images for calcified areas. Enchondroma is diagnosed on the basis of histological findings, but typical enchondroma without symptoms does not require histological examination. Surgical resection is the mainstay of treatment for this tumor, and curettage with or without bone grafting is commonly performed in patients with painful enchondromas (5-7), although most enchondromas are asymptomatic, and treatment is not required. Because a large number of enchondromas are asymptomatic, the actual tumor incidence may be higher than the one reported. The metaphysis of long bone is a common site of occurrence of enchondromas, and finger bone, followed by the humerus and femur, is the most common tumor site. Enchondromas may arise as multiple lesions, with conventional multiple enchondromas including Ollier disease and Maffucci syndrome $(8,9)$. However, some multiple enchondroma cases cannot be classified into these conventional enchondromatoses. Multiple lesions are observed in some patients with enchondroma of the hand. In this retrospective study, the distribution of solitary and multiple enchondromas was investigated to identify the characteristic feature of multiple enchondromas of the hand.

\section{Patients and Methods}

This retrospective study included 118 patients (70 women and 48 men) with enchondroma of the hand who had undergone surgical treatment for the lesions between April 2004 and December 2017 at the Nagoya City University Hospital. Patients with painful lesions or pathological fractures were indicated for surgical treatment. The latter underwent 
surgery only after fracture healing. The lesions of enchondroma of the hand were treated by endoscopic curettage of the tumor without bone grafting (5). Resected tumor specimens from all study patients were histologically evaluated to confirm the diagnosis of enchondroma. In patients with multiple lesions in the hands, the lesions were considered as enchondromas if they showed similar characteristics on radiographs and MRI and the diagnosis of enchondroma was histologically confirmed in at least one of them. To assess the incidence and characteristic features of multiple enchondromas of the hand among study patients, age, gender, distribution of the tumor, and number of lesions (solitary or multiple) were investigated.

The study protocol was approved by the Medical Ethics Committee of the Nagoya City University on November 26, 2018 (IRB number 60-18-0143). All data were anonymized, and the need for informed consent was waived by the medical ethics committee.

\section{Results}

This study included 114 patients with solitary enchondromas and 4 patients with multiple enchondromas. None of the patients had Ollier disease or Maffucci syndrome. Patients with solitary enchondromas comprised 69 women and 45 men with a mean age of 36.5 years (range $=4-80$ years). In the 114 patients with solitary enchondromas, 5 lesions occurred in the thumb, 14 in the index finger, 22 in the middle finger, 32 in the ring finger, and 41 in the little finger. Solitary enchondromas occurred in 21 metacarpal bones, 57 proximal phalanges, 24 middle phalanges, and 12 distal phalanges.

Patients with multiple enchondromas comprised three men and one woman with a mean age of 15.5 years (range $=10-19$ years) years. In all these patients, three lesions successively occurred in the same digital ray. The radiograph of a 16-yearold man (case 1) showed osteolytic lesions in the proximal phalanx and metacarpal bone of the ring finger (Figure 1); MRI showed low signal intensity lesions on T1-weighted images and high signal intensity lesions on T2-weighted images in the middle phalanx, proximal phalanx, and metacarpal bone of the ring finger. The radiograph of a 19-year-old man (case 2) showed osteolytic lesions; MRI showed abnormal lesions with alteration of signal intensity in the middle phalanx, proximal phalanx, and metacarpal bone of the index finger (Figure 2). The radiograph of a 17-year-old man (case 3) showed tumor lesions in the middle phalanx, proximal phalanx, and metacarpal bone in the middle finger (Figure 3). Furthermore, the radiograph of a 10-year-old girl (case 4) showed osteolytic lesions in the proximal phalanx and metacarpal bone of the middle finger (Figure 4); MRI showed low-intensity lesions on T1-weighted images and high-intensity lesions on T2-weighted images in the middle phalanx, proximal phalanx, and metacarpal bone of the middle finger.

\section{Discussion}

Most enchondromas are asymptomatic and detected only if they present with symptoms such as swelling, pain, and deformity. The exact incidence and distribution of this condition is difficult to investigate because most cases are latent due to lack of symptoms; however, some studies have reported the distribution of enchondromas. Reportedly, solitary enchondromas have a predilection for ulnar-sided tubular bones $(2,10)$. The proximal phalanges are most frequently affected, followed by the middle phalanges, metacarpals, and distal phalanges (11). In the present study, solitary enchondromas showed a predilection for the ulnarsided bones, consistent with previous reports.

In all four patients with multiple enchondromas of the hand, the lesions successively occurred in the same digital ray. There are two hypotheses explaining the occurrence of multiple lesions in consecutive bones. One possible explanation is the accidental consecutive occurrence of the lesions; however, the probability of the three lesions occurring consecutively is very low, and the probability that this happened in four patients is extremely low. Another hypothesis is that the occurrence of the lesions preceded the separation of the phalanges and metacarpal bones. The sequence of upper limb development in the human embryo may explain the distribution of multiple enchondromas of the hand (Figure 5) (12). The limb bud appears on intrauterine day 26 and the hand paddle on day 33. On day 36, chondrification of the humerus, radius, and ulna occurs. On day 41 , the digital rays are evident within the hand paddle and chondrification of the metacarpal bone occurs. On day 44 , chondrification occurs in the proximal phalanges. On day 47 , chondrification of the middle phalanges, initial separation of the fingers, and joint interzones are apparent in the hand. On day 50, chondrification of the proximal parts of the distal phalanges occurs. Separation of the finger bones occurs from day 47 onwards and is completed on day 54. If a tumor lesion arises before intrauterine day 41, it may get separated with the chondrification of finger bones during limb development (between days 41 and 54). In the present study, the successive occurrence of multiple enchondromas in a digital ray suggests that the lesions had been present before the separation of the finger bones was completed (Figure 6). Based on their characteristic distribution, multiple enchondromas can be classified into two types: one type comprises conventional enchondromatosis including Ollier disease and Maffucci syndrome, which independently occur in several bones; the other type comprises multiple enchondromas originally occurring as one lesion before intrauterine day 54 and subsequently separating by the chondrification of the phalanges and metacarpals during limb development in the human embryo.

The present study has some limitations including its small sample size. To diagnose enchondroma on the basis of pathological evaluation, this study included only those patients who underwent surgical resection of the tumor. Additionally, the inclusion criteria may have influenced the 


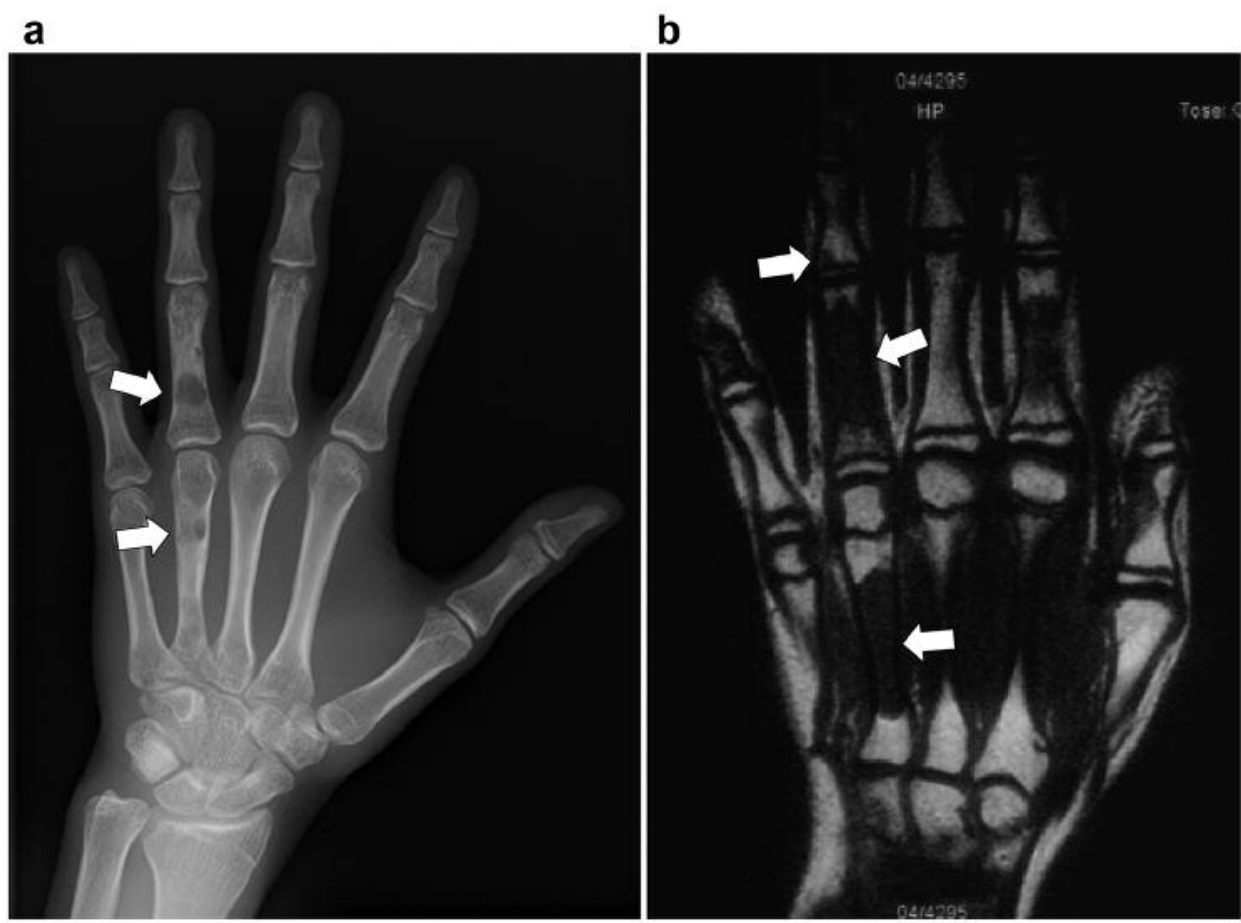

Figure 1. Case 1. A 16-year-old male patient. Radiograph showing osteolytic lesions in the proximal phalanx and metacarpal bone of the ring finger (a). Magnetic resonance imaging (MRI) showing multiple lesions in the middle phalanx, proximal phalanx, and metacarpal bone (b).
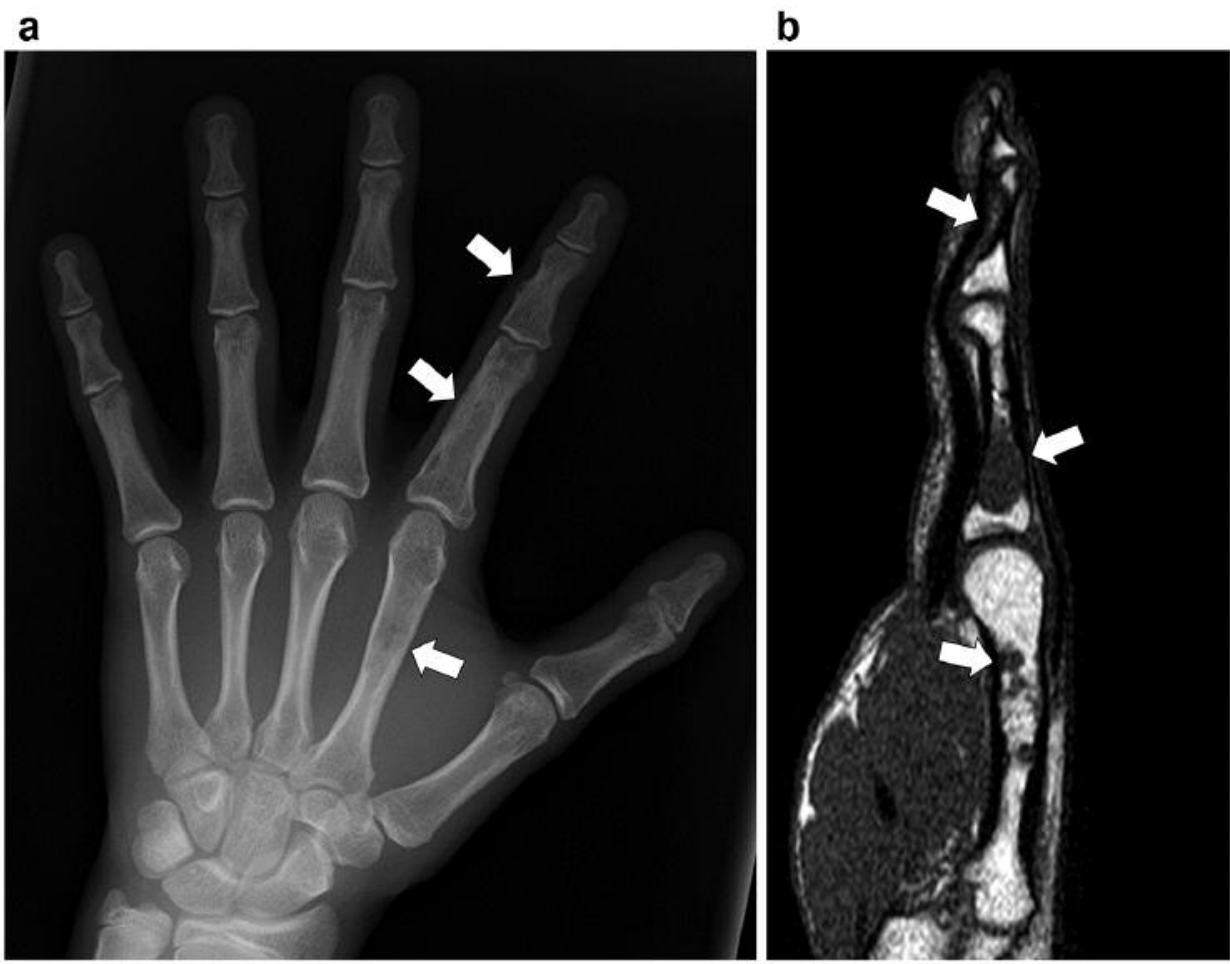

Figure 2. Case 2. A 19-year-old male patient. Radiograph (a) and MRI (b) showing abnormal lesions in the middle phalanx, proximal phalanx, and metacarpal bone of the index finger. 


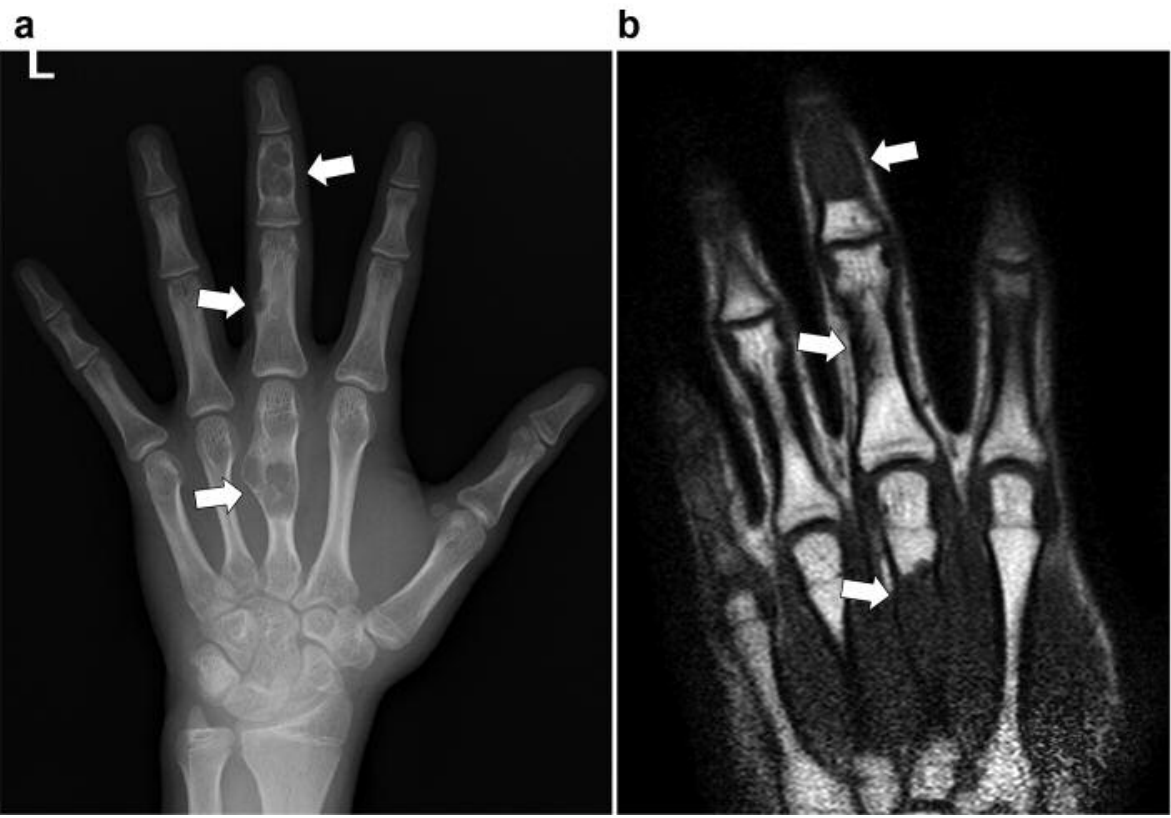

Figure 3. Case 3. A 17-year-old male patient. Radiograph and MRI showing abnormal lesions in the middle phalanx, proximal phalanx, and metacarpal bone of the middle finger.

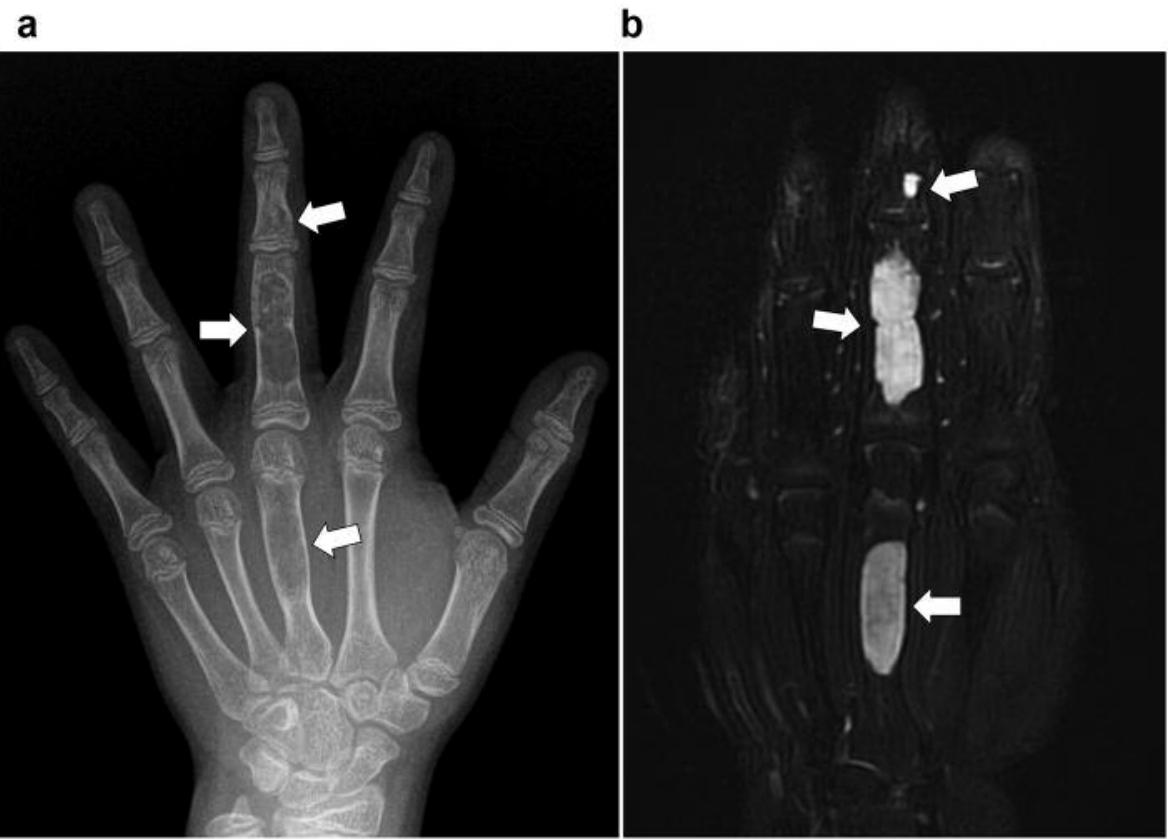

Figure 4. Case 4. A 10-year-old female patient. Radiograph showing osteolytic lesions in the proximal phalanx and metacarpal bone of the middle finger (a). MRI showing low-intensity lesions on T1-weighted images and high-intensity lesions on T2-weighted images in the middle phalanx, proximal phalanx, and metacarpal bone of the middle finger $(b)$.

exclusion of patients with Maffucci syndrome and Ollier disease. These enchondromas commonly manifest with multiple lesions in the hand, with the lesions arising in several digital rays. Common indications for surgical treatment of enchondromas include pain or pathological fracture (3), and patients with Maffucci syndrome and Ollier disease rarely undergo surgical treatment for the lesions of the hand. Further studies with a larger sample size are 

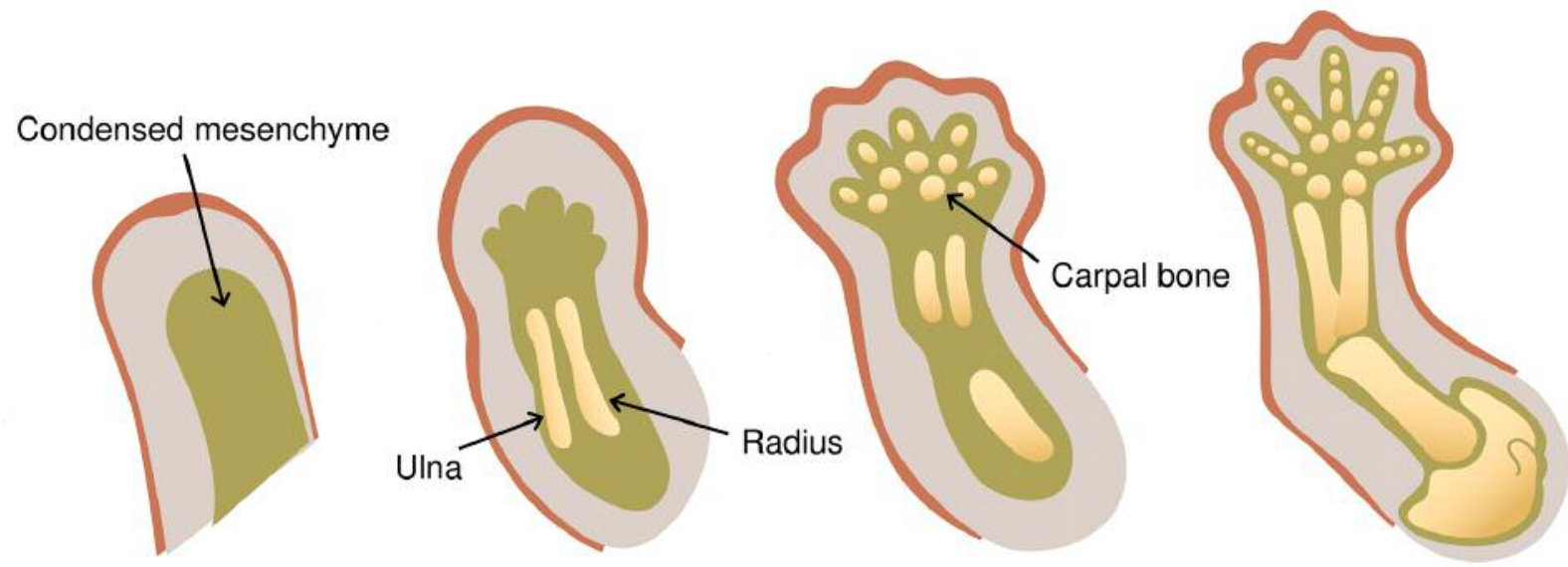

day 26

day 36

day 47

day 54

Figure 5. Hand development in the human embryo. The limb bud appears on intrauterine day 26 and hand paddle on day 33. On day 36, chondrification of the humerus, radius, and ulna occurs. On day 41, digital rays are evident within the hand paddle. On day 47, chondrification of the middle phalanges, initial separation of the finger bones, and joint interzones are apparent in the hand. On day 50, chondrification of the proximal parts of the distal phalanges occurs. On day 54, finger bones are completely separated.

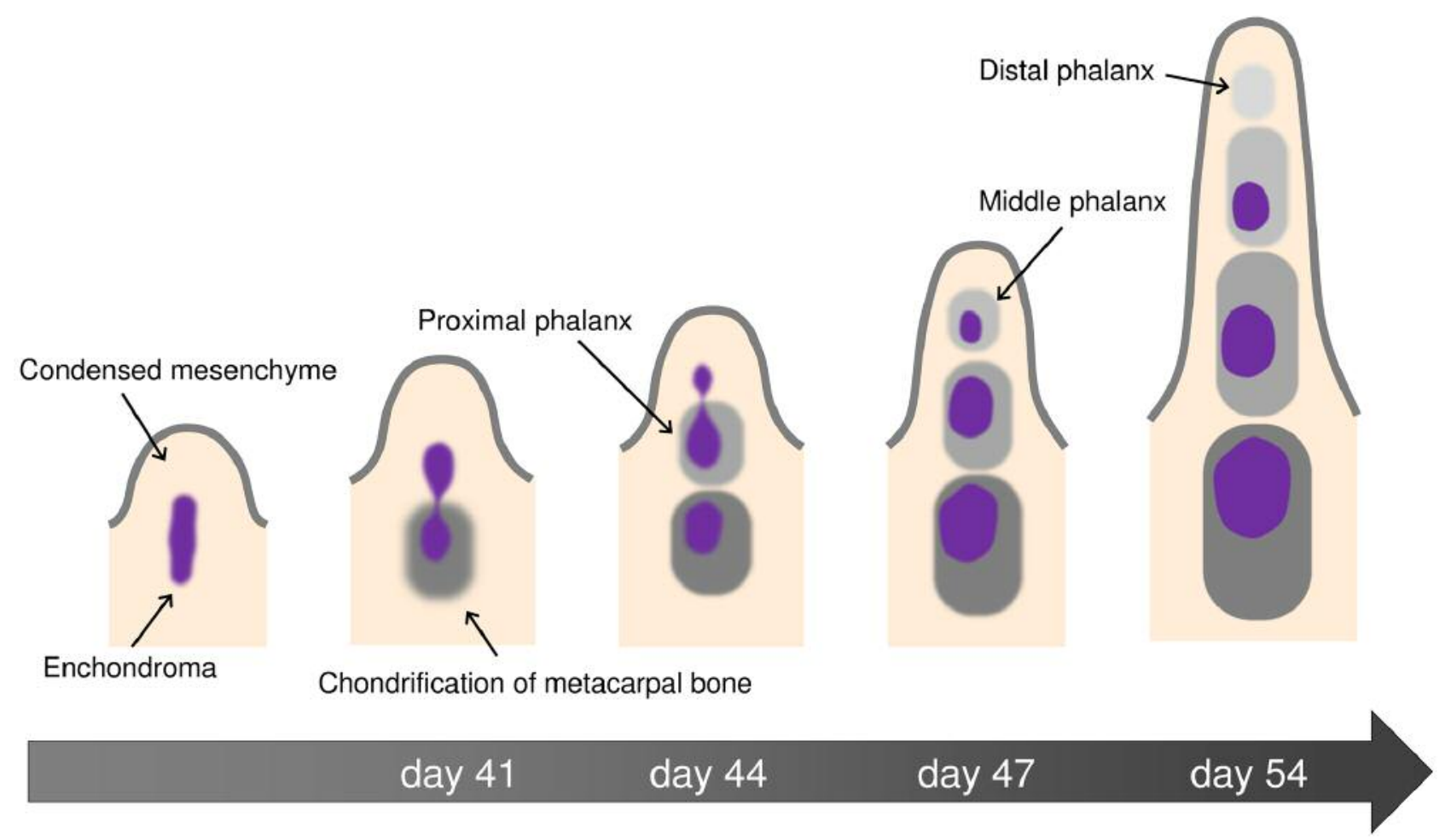

Figure 6. Occurrence of multiple enchondromas during hand development. For patients with multiple enchondromas occurring successively in the same digital ray, it is hypothesized that the lesions preceded the chondrification of the phalanges and metacarpals. If an enchondroma occurs before the development of phalanges and metacarpals, it can be separated by chondrification around the lesion during limb development, and the divided lesions can grow in separate bones as multiple enchondromas. 
required to confirm the characteristic features of multiple enchondromas of the hand.

\section{Conclusion}

The present study reported the characteristic features of multiple enchondromas in four $(3.4 \%)$ of 118 patients with enchondromas of the hand. In each case, the enchondromas successively occurred in the same digital ray. The authors consider that the tumor in the digital ray might have partially occurred before intrauterine day 54 and that the lesions separated during the development of the hand. This is the first study reporting the time of tumor occurrence according to the characteristic distribution of bone tumors.

\section{Conflicts of Interest}

The Authors declare that they have no competing interests regarding this study.

\section{Authors' Contributions}

Conceptualization: Shinji Miwa, Hideki Okamoto, Takanobu Otsuka, Hiroyuki Tsuchiya; Data curation: Shinji Miwa, Hideki Okamoto, Satoshi Yamada, Hisaki Aiba, Yohei; Kawaguchi, Kojiro Endo, Katsuhiro Hayashi, Hiroaki Kimura; Formal analysis: Shinji Miwa, Hideki Okamoto; Funding acquisition: Shinji Miwa; Investigation: Shinji Miwa, Hideki Okamoto, Satoshi Yamada, Hisaki Aiba, Yohei; Kawaguchi, Kojiro Endo, Katsuhiro Hayashi, Hiroaki Kimura; Methodology: Shinji Miwa, Hideki Okamoto, Takanobu Otsuka, Hiroyuki Tsuchiya; Project administration: Shinji Miwa, Hideki Okamoto, Satoshi Yamada, Hisaki Aiba, Yohei Kawaguchi, Kojiro Endo, Katsuhiro Hayashi, Hiroaki Kimura, Takanobu Otsuka, Hiroyuki Tsuchiya; Resources: Shinji Miwa; Supervision: Hideki Okamoto, Takanobu Otsuka, Hiroyuki Tsuchiya; Validation: Shinji Miwa, Hideki Okamoto, Takanobu Otsuka, Hiroyuki Tsuchiya; Visualization: Shinji Miwa; Writingoriginal draft: Shinji Miwa, Hideki Okamoto, Takanobu Otsuka, Hiroyuki Tsuchiya; Writing-review \& editing: Shinji Miwa, Hideki Okamoto, Satoshi Yamada, Hisaki Aiba, Yohei Kawaguchi, Kojiro Endo, Katsuhiro Hayashi, Hiroaki Kimura, Takanobu Otsuka, Hiroyuki Tsuchiya.

\section{References}

1 Shimizu K, Kotoura $\mathrm{Y}$, Nishijima $\mathrm{N}$ and Nakamura $\mathrm{T}$ : Enchondroma of the distal phalanx of the hand. J Bone Joint Surg Am 79(6): 898-900, 1997. PMID: 9199388. DOI: 10.2106/00004623-199706000-00014
2 Gaulke R: The distribution of solitary enchondromata at the hand. J Hand Surg Br 27(5): 444-445, 2002. PMID: 12367543.

3 Lubahn JD and Bachoura A: Enchondroma of the Hand: Evaluation and Management. J Am Acad Orthop Surg 24(9): 625-633, 2016. PMID: 27454024. DOI: 10.5435/JAAOS-D-1500452

4 Jewusiak EM, Spence KF and Sell KW: Solitary benign enchondroma of the long bones of the hand. J Bone Joint Surg Am 53(8): 1587-1590, 1971. PMID: 4941379.

5 Sekiya I, Matsui N, Otsuka T, Kobayashi M and Tsuchiya D: The treatment of enchondromas in the hand by endoscopic curettage without bone grafting. J Hand Surg Br 22(2): 230-234, 1997. PMID: 9149995. DOI: 10.1016/s0266-7681(97)80070-8

6 Zhou X, Zhao B, Keshav P, Chen X, Gao W and Yan H: The management and surgical intervention timing of enchondromas: A 10-year experience. Medicine (Baltimore) 96(16): e6678, 2017. PMID: 28422880. DOI: 10.1097/MD.0000000000006678.

7 Lin SY, Huang PJ, Huang HT, Chen CH, Cheng YM and Fu YC: An alternative technique for the management of phalangeal enchondromas with pathologic fractures. J Hand Surg Am 38(1): 104-109, 2013. PMID: 23200218. DOI: 10.1016/j.jhsa.2012.08.045

8 Kumar A, Jain VK, Bharadwaj M and Arya RK: Ollier disease: pathogenesis, diagnosis, and management. Orthopedics 38(6): e497-506, 2015. PMID: 26091223. DOI: 10.3928/0147744720150603-58

9 Herget GW, Strohm P, Rottenburger C, Kontny U, Krauß T, Bohm J, Sudkamp N and Uhl M: Insights into enchondroma, enchondromatosis and the risk of secondary chondrosarcoma. Review of the literature with an emphasis on the clinical behaviour, radiology, malignant transformation and the follow up. Neoplasma 61(4): 365-378, 2014. PMID: 24645839. DOI: 10.4149/neo_2014_046

10 Takigawa K: Chondroma of the bones of the hand. A review of 110 cases. J Bone Joint Surg Am 53(8): 1591-1600, 1971. PMID: 5121800.

11 Sassoon AA, Fitz-Gibbon PD, Harmsen WS and Moran SL: Enchondromas of the hand: factors affecting recurrence, healing, motion, and malignant transformation. J Hand Surg Am 37(6): 1229-1234, 2012. PMID: 22542061. DOI: 10.1016/j.jhsa. 2012.03.019

12 Al-Qattan MM, Yang Y and Kozin SH: Embryology of the upper limb. J Hand Surg Am 34(7): 1340-1350, 2009. PMID: 19700076. DOI: 10.1016/j.jhsa.2009.06.013
Received August 8, 2019

Revised September 11, 2019 Accepted September 12, 2019 